\title{
Salinidad y temperatura óptimas para reproducción ovípara y desarrollo de Artemia franciscana
}

\author{
Salinity and temperature optimum for oviparous reproduction \\ and development of Artemia franciscana
}

\author{
Paola Moraga C. , Roberto Ávila P. ${ }^{1}$, Arnaldo Vilaxa $O .^{1}$
}

\section{RESUMEN}

Artemia franciscana es uno de los organismos vivos más utilizados para la alimentación de especies en cultivo, debido a que presenta varias proteínas y ácidos grasos insaturados que juegan un rol fundamental en diversas funciones metabólicas de las especies cultivadas. Esta especie corresponde a un crustáceo pequeño branquiópodo, filtrador no selectivo, propio de hábitats acuáticos de elevada salinidad. En la etapa adulta pueden ser especies bisexuales y partenogenéticas, en ambas poblaciones las hembras pueden dar lugar a dos tipos de reproducción, ovovivípara que resulta en un nauplio completamente formado; y ovípara que resulta un cistos que pueden dar una eclosión inmediata o entrar en estado criptobiótico o de diapausa por largos períodos, facilitando la obtención de nauplios durante todo el año.

Con el objetivo de establecer las variables de salinidad y temperatura óptimas para la reproducción ovípara y posterior desarrollo de Artemia franciscana, en la zona norte de Chile, se realizó un cultivo experimental con adultos reproductores, los que fueron sometidos a nueve tratamientos con tres réplicas, a diferentes rangos de salinidad $(100 \mathrm{~g} / \mathrm{L}, 120 \mathrm{~g} / \mathrm{L}$ y $140 \mathrm{~g} / \mathrm{L})$ y temperatura $\left(20^{\circ} \mathrm{C}, 25^{\circ} \mathrm{C}\right.$ y $\left.30^{\circ} \mathrm{C}\right)$, donde los cistos obtenidos se colectaron diariamente y se almacenaron durante dos semanas para después determinar la calidad de estos sobre la base del porcentaje y eficiencia de eclosión, aplicándose a los datos obtenidos un análisis de varianza de dos vías (ANOVA) para establecer diferencias significativas entre los tratamientos.

Los resultados del análisis establecieron que existe diferencia significativa entre la salinidad y temperatura de experimentación, debido a que por separado solamente la salinidad presenta una incidencia en la reproducción. Se pone de manifiesto que los parámetros fisicoquímicos adecuados para reproducción ovípara de A. franciscana es de $120 \mathrm{~g} / \mathrm{L}$ a $25^{\circ} \mathrm{C}$, debido a que presenta una diferencia significativa al nivel $\mathrm{p}<0,054$.

Palabras clave: Artemia, cistos, criptobyosis, reproducción ovípara.

\begin{abstract}
Artemia franciscana is one of the most used for food crop species in living organisms, because it has a number proteins and unsaturated fatty acids that play a key role in various metabolic functions of cultured species. This species corresponds to a small crustacean branquiópodo, non-selective filter, typical of aquatic habitats of high salinity. In adulthood, they can be bisexual and parthenogenetic species, females in both populations may result two types of reproduction, and ovoviviparous resulting in a fully formed nauplii may result; resulting in an oviparous and cysts that can provide an immediate explosion or enter diapause or cryptobiotic state for long periods of time, facilitating the production of nauplii throughout the year.

In order to set the variables of salinity and optimum temperature for oviparous and subsequent development of Artemia franciscana in northern Chile, an experimental crop breeding adults, who underwent nine treatments with three replicates were performed, to different ranges of salinity $(100 \mathrm{~g} / \mathrm{L}, 120 \mathrm{~g} / \mathrm{L}$ and $140 \mathrm{~g} / \mathrm{L})$ and temperature $\left(20^{\circ} \mathrm{C}, 25^{\circ} \mathrm{C}\right.$ and $\left.30^{\circ} \mathrm{C}\right)$, where the cysts obtained were collected daily and stored for two weeks and then determining their quality and based on the percentage hatching efficiency and applied to the analysis of data from two-way variance (ANOVA) to establish significant differences between treatments.

The test results established that there is significant difference between the experimental temperature and salinity because salinity separately only has an effect on reproduction. Is underlined that adequate physicochemical parameters for oviparous A. franciscana is $120 \mathrm{~g} / \mathrm{L}$ at $25^{\circ} \mathrm{C}$ because it presents a significant difference at $p<0.054$ level.
\end{abstract}

Key words: Artemia, cystos, criptobyiosis, oviparous reproduction.

\footnotetext{
1 Centro de Estudios Marinos y Limnológicos, Universidad de Tarapacá, Facultad de Ciencias, Arica, Chile.

* Autor por correspondencia: avilaxa@uta.cl
}

Fecha de Recepción: 2 Agosto, 2014.

Fecha de Aceptación: 14 Noviembre, 2014. 


\section{Introducción}

La acuicultura es una actividad que hace posible el cultivo y producción de peces, moluscos, algas y crustáceos en una gran diversidad de ambientes acuáticos controlados, siendo una actividad económica con un alto potencial para generar trabajo de bajo impacto en el ambiente y altas divisas para cualquier región del mundo (Avilés y Castelló, 2004). En este sentido el alimento es uno de los factores más importantes en el cultivo de peces marinos, ya que su conocimiento y elección representan un alto porcentaje en el gasto operativo de las empresas (Avilés y Castelló, 2004). Sin embargo, durante los últimos veinte años se han desarrollado dietas artificiales y la mayoría de los estudios han indicado un crecimiento y supervivencia inferior al alimento vivo (Lazo, 2000; Castro et al., 2003; Villamar, 2004; Luna- Figueroa et al., 2010).

De los organismos vivos más utilizados para la nutrición de peces se encuentran copépodos, rotíferos y artemias (Sánchez-Estudillo, 2010), la utilización de este último comienza en el estadio naupliar y en la actualidad es el microcrustáceo vivo más empleado para la alimentación de especies en cultivo (Castro et al., 2003; Luna-Figueroa al., 2010; ), debido a que presenta proteínas y lípidos que incluyen ácidos grasos insaturados, los que juegan un papel importante en diversas funciones metabólicas de los organismos en cultivo (Martínez et al., 2004; Zamora y Shpigel, 2006), estas características han permitido que tengan un alto valor comercial y que el consumo de cistos se encuentre por las 1.700 toneladas anuales. (Sorgeloos et al., 1991; Castro et al., 1997; Esteve y El Masri, 2007).

El 90\% de los cistos que se consumen a nivel mundial provienen del Gran Lago Salado de Utah, Estados Unidos; sin embargo, cambios climáticos que ha sufrido el medio ambiente a nivel mundial ha hecho necesario buscar nuevas formas de abastecimiento que puedan suplir las demandas de mercados nacionales e internacionales (Lavens y Sorgeloos, 2000; Sato et al., 2004), lo que se complica por el hecho de que aún no se tiene claro cuáles son los factores que inducen a la formación de cistos, los que tienen las características de presentar resistencia a los factores abióticos del ambiente (De los Ríos-Escalante y Salgado, 2012) y pueden permanecer en un estado de latencia por muchos años (mientras permanezcan secos), estado conocido como criptobiosis (Clegg, 2001). Estos cistos una vez que las condiciones se vuelven favorables eclosionan y continúan con su ciclo de vida hasta la etapa adulta.

Debido a que la principal fuente de abastecimiento de cistos en el mundo está bajo la incertidumbre del aporte futuro que pueda hacer a los cultivos, se hace imperioso establecer una técnica que permita disponer de cistos durante todo el año, pudiendo producirlos y almacenarlos.

La ventaja de contar con cistos es que estos son resistentes a condiciones adversas y porque una vez dadas las condiciones para su descapsulación, presentan tasas de crecimiento mayor en relación con nauplios sin decapsular (Rodríguez-Canché et al., 2006); y considerando la alta demanda de este recurso en la alimentación de especies en cautiverio, es que el objetivo de la presente investigación fue establecer las variables ambientales óptimas para la reproducción ovípara y posterior desarrollo de $A$. franciscana, y con ello lograr un alimento óptimo de bajo costo con disminución de la dependencia de cistos foráneos.

\section{Materiales y Métodos}

Los cistos de Artemia franciscana fueron obtenidos desde Utah, EE.UU., de la empresa Biomarine, INC.-Aquafauna, y para el cultivo experimental se utilizó el laboratorio de Biología del Desarrollo de la Universidad de Tarapacá.

La primera etapa del trabajo consistió en la descapsulación de cistos, para ello se pesó $1 \mathrm{~g}$ de cistos y se colocó en $100 \mathrm{~mL}$ de una solución de hipoclorito de sodio comercial y agua de mar filtrada en una proporción 1:2; una vez que los cistos tomaron una coloración anaranjada intensa se tamizaron en una red para plancton de 20 micrones y se dejaron por una hora en un flujo suave y constante de agua corriente para eliminar el exceso de hipoclorito de sodio, finalizado el proceso se enjuagaron con agua de mar filtrada.

La eclosión se realizó en una incubadora artesanal, esta consistió en un recipiente de plástico negro con una ventana en uno de sus costados con $500 \mathrm{~mL}$ de agua de mar filtrada a una temperatura que osciló entre $24-30{ }^{\circ} \mathrm{C}$ y pH 8 , que estuvo bajo aireación constante. Una vez ocurrida la eclosión se retiró la aireación, y se acercó la luz a la ventana del incubador durante una hora, para aprovechar el fototropismo positivo, y realizar la colecta de los nauplios mediante sistema de sifoneo. 
Para el cultivo los nauplios se trasladaron a un recipiente para su crecimiento, estableciendo una densidad de cultivo de 100 nauplios/500 $\mathrm{mL}$. En cuanto a su alimentación, se dividió en dos etapas, la naupliar y la adulta; durante la etapa naupliar se alimentaron con una dosis diaria de $15 \mathrm{~mL}$ de cultivo de Chlorella sp., y en la etapa adulta se alimentaron con una dosis diaria de $15 \mathrm{~mL}$ de una solución de levadura con leche en una relación 1:1, esto debido a que la leche proporciona una serie de nutrientes como proteínas, lípidos y carbohidratos. En ambos casos la alimentación se aplicaba por las mañanas, posterior a ello se esperó dos horas y luego se realizó el recambio de agua.

El cultivo de A. franciscana fue multifactorial, para ello se utilizó nueve recipientes por triplicado, los que fueron sometidos a distintas temperaturas, 20,25 y $30^{\circ} \mathrm{C}$, a cada una de ellas se le agregó una concentración salina de 100, 120 y 140 g/L, para luego introducir en cada recipiente una población de 80 reproductores ( 40 machos y 40 hembras); el resto de las variables como el oxígeno, la cantidad de alimento y la luminosidad se mantuvieron constantes.

La recolección de cistos se realizó con una pipeta de vidrio de $10 \mathrm{~mL}$, los que se depositaron en una cápsula Petri y se contaron bajo lupa, una vez realizado el conteo fueron almacenados en salmuera a una concentración de $35 \mathrm{~g} / \mathrm{L}$, hasta completar dos semanas. Después de aquel tiempo los cistos fueron lavados para eliminar los restos de sal, centrifugados para eliminar los cistos no viables y colocados en una superficie plana con papel filtro para su secado. Una vez que terminó el secado, que duró 24 horas, los cistos se almacenaron durante dos semanas, entre 24 y $25^{\circ} \mathrm{C}$, en tubos de ensayo de $25 \mathrm{~mL}$ de capacidad, los tubos fueron rotulados, forrados en papel metálico y taponado, para evitar la luminosidad, el exceso de humedad y los cambios de temperatura.

Para evaluar la calidad de los cistos se masaron 0,001 gramos y se determinó de manera manual la cantidad de cistos existentes en dicho valor, el tamaño se determinó midiendo el diámetro de cada uno de estos, bajo microscopia mediante el software Micrometric SE premiun. Se realizaron 30 ensayos y se estableció un promedio de estos.

La segunda etapa fue determinar la eficiencia de eclosión (EE), porcentaje de eclosión (PE) y la tasa de eclosión (TE), para este procedimiento se utilizaron las técnicas de eclosión antes mencionadas.
Para el análisis estadístico entre los distintos tratamientos se utilizó el programa SPSS17 Inc. La cuantificación de cistos producidos por A. franciscana en los tratamientos se determinó por medio de un análisis de ANOVA univariado (GML) a un nivel de significancia del 0,05 , donde se consideró la variable respuesta a la producción de cistos versus variables fijas, temperatura y salinidad.

Posteriormente se realizó un análisis posthoc para las medias observadas de Tukey y DMS, se compararon los efectos principales de las medias marginales de acuerdo con el ajuste de intervalo de confianza de Bonferroni para generar (overall), salinidad, temperatura y para salinidad-temperatura y finalmente se realizó un análisis de homogeneidad.

\section{Resultados y Discusión}

Los cistos de A. franciscana utilizados en el estudio, según rotulado, presentan $90 \%$ de eclosión, en tanto que en esta investigación el porcentaje de eclosión fue de $84 \%$ (Tabla 1). Lavens y Sorgeloos (2000) y Sato et al. (2004) señalan que en ocasiones los cambios medioambientales influencian en el estado de criptobiosis, aminorando la tasa de eclosión, lo que puede atribuirse al caso, ya que se desconocen las condiciones en que se determinó dicho porcentaje con relación a las variables sometidas en esta investigación, si bien tampoco fue considerable la baja. Se puede desprender que mientras más años se encuentren en criptobiosis los cistos, menor será su porcentaje de eclosión. Rodríguez-Canché et al., 2006, y Eslava-Eljaiek et al., 2011, también manifiestan que el pobre porcentaje de eclosión de los quistes se puede deber también a las diferencias genéticas entre las poblaciones, factores climáticos y fisicoquímicos del ambiente, técnicas de limpieza y procesado del quiste; por tal motivo, el hecho de haber utilizado hipoclorito de sodio para su descapasulación no se descarta que también haya

Tabla 1. Porcentaje de eclosión de tres muestras de cistos de Artemia.

\begin{tabular}{cc}
\hline Muestras & Porcentaje (\%) \\
\hline M 1 & $73,00 \%$ \\
M 2 & $94,00 \%$ \\
M 3 & $87,00 \%$ \\
\hline Promedio & $\mathrm{X}=84,67 \% \pm 8,73$ \\
\hline
\end{tabular}


incidido en la eclosión, situación que puede dar pie para una nueva investigación científica.

En relación con el cultivo, en los nueve tratamientos las hembras presentaron sus primeros cistos a los cinco días de haber copulado. Como se muestra en la Figura 1, el índice de mortalidad varió en los distintos tratamientos.

En los tres tratamientos el promedio de cistos varió dependiendo de la temperatura. En la población cultivada a $100 \mathrm{~g} / \mathrm{L}$ a $20^{\circ} \mathrm{C}$ se cosecharon 183 cistos, a $25^{\circ} \mathrm{C} 162$ cistos y finalmente a los $30{ }^{\circ} \mathrm{C}$ la cosecha fue de 134 cistos. Mientras que en la población cultivada a $120 \mathrm{~g} / \mathrm{L}$ de $\mathrm{NaCl}$ a una temperatura de $20{ }^{\circ} \mathrm{C}$, la cosecha fue de 158 individuos, en $25^{\circ} \mathrm{C}$ se presentó la cohecha más abundante promediando 196 cistos, mientras que en la población cultivada a $30^{\circ} \mathrm{C}$ existió una cosecha de 122 cistos. En la población cultivada a 140 g/L en los tres tratamientos la mortalidad fue $100 \%$.

En cuanto al desarrollo hasta la etapa nauplios, se observó que los nauplios seguían los parámetros establecidos por Malpica et al., 1997 y Cisneros y Vinatea 2009, promediando 25 días antes de la etapa adulta, situación que puede manifestarse básicamente por los elevados valores proteicos y lipídicos del alga Chlorella sp. (Morris et al., 1999, Infante et al., 2012). Otros estudios, en la segunda etapa, el alimento se basó en una mezcla de levadura con leche; observando un reducción en los índices de mortalidad, situación que se respalda por lo manifestado por Esteve y El Marsi, 2007, quienes señalan que la alimentación con levadura provoca menores índices de mortalidad que las dietas a base de harina de algas y produce un aumento en la reproducción ovípara de las especies. El criterio para la elección de la leche se basó en su aporte nutricional.

La cantidad de cistos por gramos depende de la subespecie de Artemia que se trate y de los parámetros fisicoquímicos a los cuales sean sometidos, en el presente estudio la cantidad de cistos por gramo osciló entre 104.000 y 178.000 (Tabla 2), valores similares a los que rescató Sato, et al. (2004), quien señala al número de cistos por gramo de muestra seca de $A$. persimilis varía entre 102.600 y 276.000 , en A. franciscana varía entre 70.000 y 110.000 , información corroborada por Luna-Figueroa, 2009, quien señala que el número de cistos por gramo varía dependiendo de la especie y del lugar donde se encuentren.

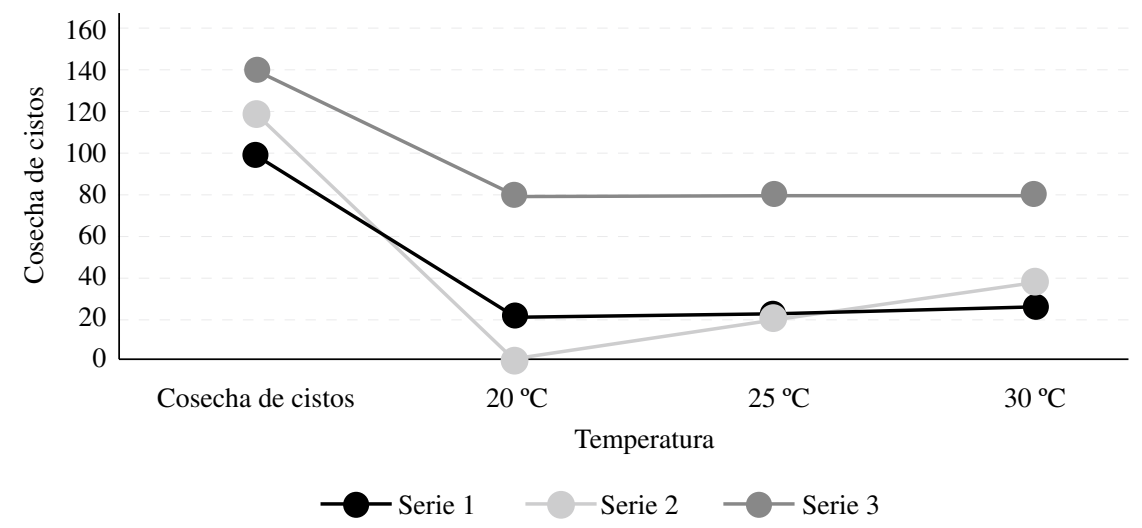

Figura 1: Mortalidad de especímenes a diferentes salinidades.

Tabla 2. Registro de la cantidad de cistos en condiciones de variación de temperatura y salinidad.

\begin{tabular}{lccc}
\hline Muestras & $\begin{array}{c}\text { Promedio de cistos en } \\
0,001 \text { gramos. } \\
100(\mathrm{~g} / \mathrm{L})\end{array}$ & $\begin{array}{c}\text { Promedio de cistos en } \\
0,001 \text { gramos. } \\
120(\mathrm{~g} / \mathrm{L})\end{array}$ & $\begin{array}{c}\text { Promedio de cistos en } \\
0,001 \text { gramos. } \\
140(\mathrm{~g} / \mathrm{L})\end{array}$ \\
\hline M 1 $\left(20^{\circ} \mathrm{C}\right)$ & 125 & 133 & 0 \\
M 2 $\left(25^{\circ} \mathrm{C}\right)$ & 137 & 178 & 0 \\
M 3 $\left(30^{\circ} \mathrm{C}\right)$ & 104 & 152 & 0 \\
\hline
\end{tabular}


Con relación a la eficiencia de eclosión, en la Figura 2 se observa que con $120 \mathrm{~g} / \mathrm{L}$ y una temperatura de $25{ }^{\circ} \mathrm{C}$ la eficiencia de eclosión resultó ser más alta y la menor eficiencia fue con $100 \mathrm{~g} / \mathrm{L}$ y a una temperatura de $30{ }^{\circ} \mathrm{C}$, como también a salinidad de $140 \mathrm{~g} / \mathrm{L}$, debido a que los individuos murieron a los pocos días de iniciada la experimentación.

Según Quynth et al., 1988, Luna-Figueroa et al., 2009, los porcentajes de eclosión de Artemia superiores al $70 \%$ son considerados como aceptables y señalan que en el mercado internacional se comercializa con quistes que tienen el $50 \%$ de eclosión o inferior. De los resultados obtenidos podemos inferir que al comparar ambas salinidades el porcentaje de eclosión más eficiente fue de $95 \%$ y ocurrió a los $120 \mathrm{~g} / \mathrm{L}$ y temperatura a $25^{\circ} \mathrm{C}$. Esto no difiere de lo señalado para cistos cosechados por Cisneros y Vinatea (2009), quienes manifiestan que el mejor porcentaje de eclosión ocurre entre los 25 a $30^{\circ} \mathrm{C}$ y que es más viable realizar la eclosión utilizando agua de mar con salinidad normal.
Se observa en las Figuras 3 y 4 una sincronía de 8 horas. Dejando de manifiesto que el mejor porcentaje de eclosión se obtuvo a los $25^{\circ} \mathrm{C}$ a una salinidad de $120 \mathrm{~g} / \mathrm{L}$.

El análisis de varianza (ANOVA) mostró diferencias significativas $(\mathrm{p}<0,05)$ entre la interacción temperatura y salinidad (Tabla 3), lo que indica que tanto la salinidad como la temperatura en conjunto son los factores de estrés que propician la reproducción ovípara, principalmente por la acción de la sal. Mientras que por separado tanto temperatura (Tabla 4) como salinidad (Tabla 5) no presentan diferencias significativas $(p>0,05)$.

En cuanto al efecto de concentración de salinidad en la reproducción ovípara, la investigación arrojó que no existen diferencias significativas entre 100 y $120 \mathrm{~g} / \mathrm{L}(\mathrm{p}=0,583)$; el tratamiento que arrojó la mayor cantidad de cistos fue el cultivado a $120 \mathrm{~g} / \mathrm{L}$, situación que se contrapone con las investigaciones realizadas por Soniraj (2004) y Castro (2010), quienes manifiestan que las especies bisexuales
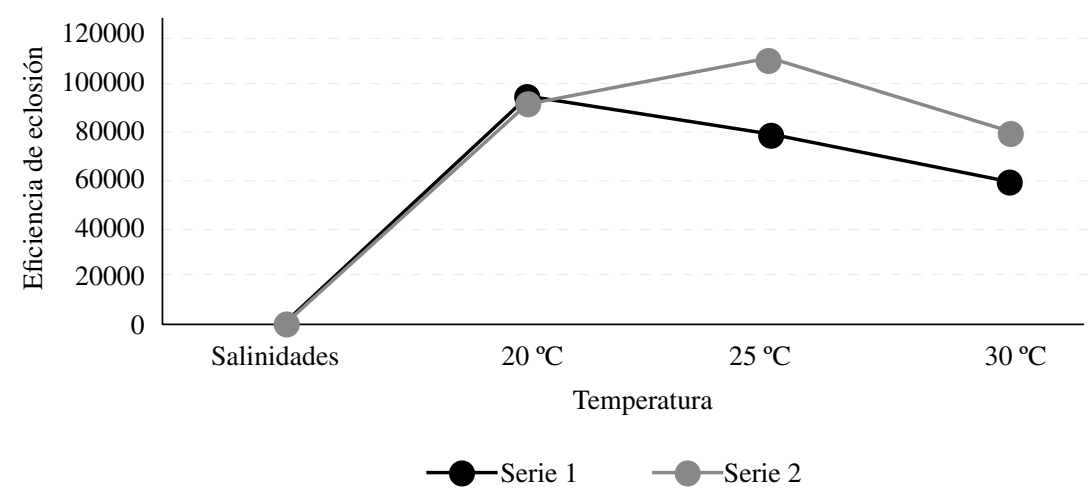

Figura 2: Eficiencia de eclosión a distintas salinidades.

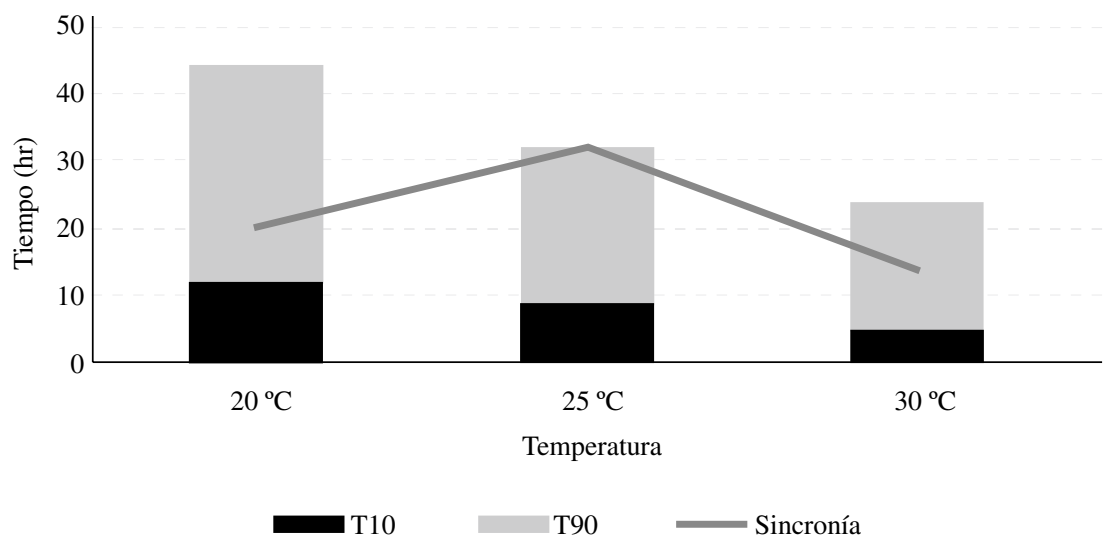

Figura 3. Tasa de eclosión a los 100 g/L. 


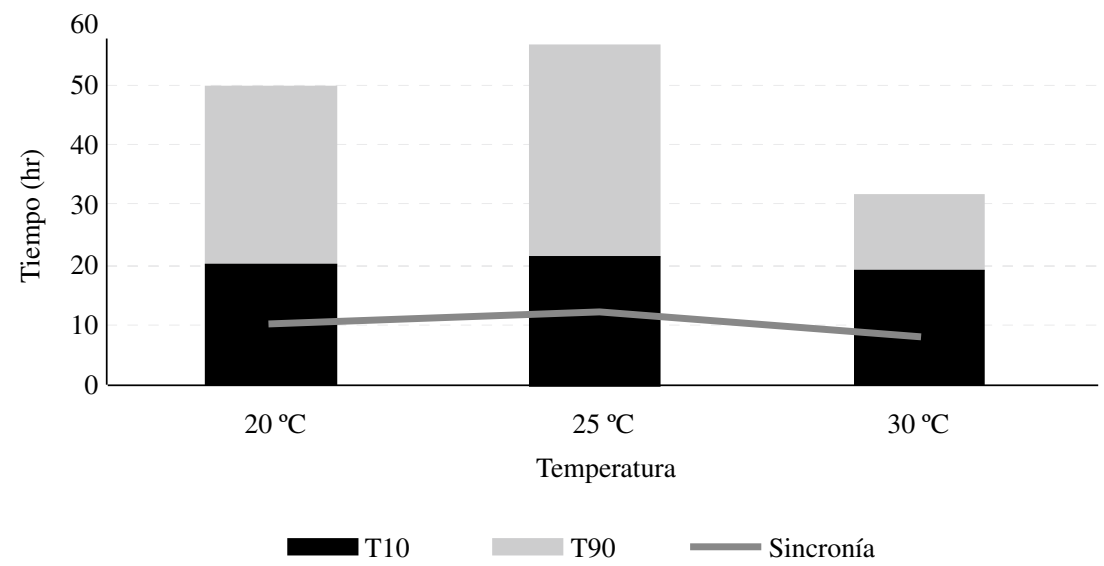

Figura 4. Tasa de eclosión a los $120 \mathrm{~g} / \mathrm{L}$.

Tabla 3. Pruebas de los efectos intersujetos. Variable dependiente: cistos producidos.

\begin{tabular}{lccccc}
\hline Origen & Suma de cuadrados tipo III & gl & Media cuadrática & F & Sig. \\
\hline Temperatura & 194952,333 & 2 & 97476,167 & 16,470 & 0,000 \\
Salinidad & 1880,889 & 1 & 1880,889 & 0,318 & 0,583 \\
Temperatura-Salinidad & 89203,444 & 2 & 44601,722 & 7,536 & 0,008 \\
& \multicolumn{2}{c}{ aR cuadrado $=0,801$ ( Rcorregida $=0,718)$} & & \\
\hline
\end{tabular}

Tabla 4. Comparaciones por pares. Variable dependiente: cistos producidos.

\begin{tabular}{|c|c|c|c|c|c|c|}
\hline \multirow[t]{2}{*}{ (I)Temperatura } & \multirow[t]{2}{*}{ (J)Temperatura } & \multirow{2}{*}{$\begin{array}{c}\text { Diferencia } \\
\text { de medias (I-J) }\end{array}$} & \multirow[t]{2}{*}{ Error típ. } & \multirow[t]{2}{*}{ Sig. ${ }^{a}$} & \multicolumn{2}{|c|}{$\begin{array}{c}\text { Intervalo de confianza al 95\% } \\
\text { para la diferencia }{ }^{\mathrm{a}}\end{array}$} \\
\hline & & & & & Límite inferior & Límite superior \\
\hline \multirow{2}{*}{$20{ }^{\circ} \mathrm{C}$} & $25^{\circ} \mathrm{C}$ & $-121,667$ & 44,416 & 0,054 & $-245,119$ & 1,786 \\
\hline & $30^{\circ} \mathrm{C}$ & $133,167 *$ & 44,416 & 0,033 & 9,714 & 256,619 \\
\hline \multirow{2}{*}{$25^{\circ} \mathrm{C}$} & $20^{\circ} \mathrm{C}$ & 121,667 & 44,416 & 0,054 & $-1,786$ & 245,119 \\
\hline & $30^{\circ} \mathrm{C}$ & $254,833^{*}$ & 44,416 & 0,000 & 131,381 & 378,286 \\
\hline \multirow{2}{*}{$30^{\circ} \mathrm{C}$} & $20^{\circ} \mathrm{C}$ & $-133,167 *$ & 44,416 & 0,033 & $-256,619$ & $-9,714$ \\
\hline & $25^{\circ} \mathrm{C}$ & $-254,833^{*}$ & 44,416 & 0,000 & $-378,286$ & $-131,381$ \\
\hline
\end{tabular}

Basadas en las medias marginales estimadas.

a Ajuste para comparaciones múltiples: Bonferroni.

* La diferencia de medias es significativa al nivel 0,05 .

Tabla 5. Comparaciones por pares. Variable dependiente: cistos producidos.

\begin{tabular}{|c|c|c|c|c|c|c|}
\hline \multirow[t]{2}{*}{ (I)Salinidad } & \multirow[t]{2}{*}{ (J)Salinidad } & \multirow{2}{*}{$\begin{array}{c}\text { Diferencia } \\
\text { de medias (I-J) }\end{array}$} & \multirow[t]{2}{*}{ Error típ. } & \multirow[t]{2}{*}{ Sig. ${ }^{a}$} & \multicolumn{2}{|c|}{$\begin{array}{c}\text { Intervalo de confianza al 95\% } \\
\text { para la diferencia }{ }^{\mathrm{a}}\end{array}$} \\
\hline & & & & & Límite inferior & Límite superior \\
\hline $100 \mathrm{ppt}$ & $120 \mathrm{ppt}$ & 20,444 & 36,265 & 0,583 & $-58,571$ & 99,460 \\
\hline $120 \mathrm{ppt}$ & $100 \mathrm{ppt}$ & $-20,444$ & 36,265 & 0,583 & $-99,460$ & 58,571 \\
\hline
\end{tabular}

Basadas en las medias marginales estimadas.

a Ajuste para comparaciones múltiples: Bonferroni. 
de A. franciscana y A. persimilis presentan mayor cantidad de cistos y nauplios cuando los adultos son colocados en 20-100 g/L de salinidad, y para ambas especies las hembras comienzan a arrojar quistes cuando alcanzan los 120-170 g/L.

Con la prueba de Tukey para establecer diferencias significativas entre las diferentes temperaturas se corroboró que la temperatura de $20^{\circ} \mathrm{C}$ y los rangos inferiores a esta se escapan de los óptimos de producción (Tabla 6).

Browne y Wanigasekera (2000) señalan que las especies de A. partenogenética, A. sinica y A. franciscana obtuvieron un mejor crecimiento y mejores producciones de nauplios y quistes cuando se cultivaron a $24^{\circ} \mathrm{C}$ y a $120 \mathrm{~g} / \mathrm{L}$, lo que concuerda con los resultados obtenidos en esta investigación. Por su parte, Salgado (2001) recomienda efectuar la eclosión entre 25 a $30{ }^{\circ} \mathrm{C}$, ya que bajo $25^{\circ} \mathrm{C}$ la eclosión se hace lenta y por encima de $30^{\circ} \mathrm{C}$ el metabolismo interno se detiene y se hace irreversible; sin embargo, hay que dejar de manifiesto que la morfología general es similar en todas las poblaciones de artemia (Correa y
Bückle, 1993), Sin embargo Castro (2009) señala que la fisiología y reproducción va a variar dependiendo del tipo de especie de Artemia que se trate.

\section{Conclusión}

La reproducción ovípara de Artemia franciscana se produce cuando estas son sometidas a condiciones de estrés biológico, como aumento de salinidad, variaciones de temperatura, falta y abundancia de alimento, donde la salinidad tiene la mayor relevancia seguida por la temperatura. De los resultados obtenidos se concluye que los parámetros ambientales óptimos para la reproducción ovípara y desarrollo de Artemia franciscana son someter al cultivo a una salinidad a $120 \mathrm{~g} / \mathrm{L}$ a temperatura de $25{ }^{\circ} \mathrm{C}$ con un pH 7 y con aireación constante. En tanto para la población parental se sugiere la cantidad de 80 individuos en $500 \mathrm{~mL}$ de agua, para una óptima reproducción. Con una dieta basada en Chorella sp., levadura y leche aportando 15 $\mathrm{mL}$ diarios en $500 \mathrm{~mL}$ de agua para 80 individuos.

Tabla 6: Producción a diferentes temperaturas.

\begin{tabular}{cccccrc}
\hline \multirow{2}{*}{$(\mathrm{I})$ Temperatura } & $(\mathrm{J})$ Temperatura & $\begin{array}{c}\text { Diferencia de } \\
\text { medias (I-J) }\end{array}$ & Error típ. & Sig. & \multicolumn{2}{c}{ Intervalo de confianza 95\% } \\
\cline { 3 - 7 } $20^{\circ} \mathrm{C}$ & $25^{\circ} \mathrm{C}$ & $-121,6667^{*}$ & 44,41575 & 0,044 & $-240,1618$ & $-3,1715$ \\
& $30^{\circ} \mathrm{C}$ & $133,1667^{*}$ & 44,41575 & 0,028 & 14,6715 & 251,6618 \\
$25^{\circ} \mathrm{C}$ & $20^{\circ} \mathrm{C}$ & $121,6667^{*}$ & 44,41575 & 0,044 & 3,1715 & 240,1618 \\
& $30^{\circ} \mathrm{C}$ & $254,8333^{*}$ & 44,41575 & 0,000 & 136,3382 & 373,3285 \\
$30^{\circ} \mathrm{C}$ & $20^{\circ} \mathrm{C}$ & $-133,1667^{*}$ & 44,41575 & 0,028 & $-251,6618$ & $-14,6715$ \\
& $25^{\circ} \mathrm{C}$ & $-254,8333^{*}$ & 44,41575 & 0,000 & $-373,3285$ & $-136,3382$ \\
\hline
\end{tabular}

Basadas en las medias observadas.

El término de error es la media cuadrática $($ Error $)=5918,278$.

* La diferencia de medias es significativa al nivel 0,05 .

\section{Literatura Citada}

Avilés, A.; Castelló, F.

2004. Manual para el cultivo de Seriola lalandi (Pisces: Carangidae) en Baja California sur de México. Instituto Nacional de la pesca. Dirección general de investigación en acuacultura. México, D.F. 47 pp.

Browne, R.; Wanigasekera, G.

2000. Combined effects of salinity and temperatura on survival and reproduction of five species of Artemia. Journal of Experimental Marine Biology and Ecology, 244: $29-44$.
Castro, J.; Malpica, A.; Castro, T.; De Lara, R.; Castro, G. 1997. El uso de nauplios y quistes de la población de Artemia de Oaxaca en el desarrollo de Acuicultura en la región del Istmo. Ciencia y Mar, 1: 35-38.

Castro, T.; De Lara, R.; Castro, G.; Castro, J., Malpica, A. 2003. Alimento vivo en la acuicultura. ContactoS, 48: 27-33. Castro, J.; Castro, T.; Arredondo, J.; Hernández, L.; Castro, G.; De Lara, R.; Monroy, M.

2009. La salinidad y su efecto en la reproducción del crustáceo Artemia sp. ContactoS, 73: 5-15. 
Castro, J.; Castro, T.; Arredondo, J., Hernández, L.; Castro, G.; De Lara, R.; Monroy, M.

2010. Potencial reproductivo de seis poblaciones mexicanas de Artemia franciscana Kellog 1906, cultivadas en laboratorios a 100 y 120 UPS. Biocyt, 3:145- 158.

Cisneros, R; Vinatea, E.

2009. Producción de biomasa de Artemia franciscana Kellogg 1906 utilizando diferentes dietas. Ecología Aplicada, 1: 9-14.

Clegg, J.

2001. Cryptobiosis a peculiar state of biological Organization. Comparative Biochemistry and Physiology Part B, 128: 613-624.

Correa, F.; Bückle, L.

1993. Morfología y biometría de cinco poblaciones de Artemia franciscana (Anostraca: Artemiidae). Rev. Biol. Trop., 41: 103-110.

De los Ríos-Escalante, P., Salgado, I.

2012. Artemia (Crustacea, Anostraca) in Chile: a review of basic and applied biology. Universidad Católica de Temuco. Aquat, 40: 487-496.

Eslava-Eljaiek, P., Wedler, E.; Serna-Macías, D.

2011. Caracterización y criterios de eclosión de quistes de Artemia sp. en la salina de pozos colorados (Santa Marta, Colombia). Rev. Intropica, 6: 101-108.

Esteve, M.; El Marsi, R.

2007. Survival, production and cysts quality of Artemia (Araya estrían), sucre state, Valenzuela, under combination of three diets and theree Salinites. Universidad de Oriente, Venezuela, 19: 3-13.

Infante, C.; Angulo, E.; Zárate, A.; Florez, J.; Barrios, F.; Zapata; C. 2012. Propagación de la microalga Chlorella sp. en cultivo por lote: cinética del crecimiento molecular. Avances en Ciencias e Ingeniería, 3: 159-164.

Lavens, P.; Sorgeloos, P.

2000. The history, present status and prospects of the availability of Artemia cysts for aquaculture. Aquaculture, 181: 397-403.

Lazo, J.P.

2000.Conocimiento actual y nuevas perspectivas en el desarrollo de dietas para larvas de peces marinos. Avances en Nutrición Acuícola V. Memorias del V Simposium Internacional de Nutrición Acuícola. Yucatán, México.

Luna-Figueroa J.; Soriano, M.; Figueroa. J.

2009. Calidad de quistes de Artemia franciscana (Crustacea: anostraca) y del camarón duende Streptocephalus mackini (Crustacea: anostraca) como alternativa para la alimentación inicial de larvas de peces. Mesoamericana, 13: 22-27.

Luna-Figueroa, J.; Vargas, T.; Figueroa, J.

2010. Alimento vivo en la dieta de larvas y juveniles de Pterophyllum scalare (Lichtenstein, 1823). Avances en Investigación Agropecuaria, 14: 63-72.
Malpica, A., Castro, M.J.; Castro, T.; De Lara, R.; Castro, M.G. 1997. Datos de capacidad reproductiva de Artemia franciscana, variedad Oaxaca. Ciencia y Mar, 10: 31-34.

Martínez, L., Campaña, A.; Martínez-Porchas, M. 2004. Manejo de la Productividad Natural en el Cultivo del Camarón. Avances en Nutrición Acuícola VII. Memorias del VII Simposium Internacional de Nutrición Acuícola. 16-19 noviembre. Hermosillo, Sonora, México.

Morris, H.; Quintana, M., Almarales A.; Hernández. L.

1999. Composición bioquímica y evaluación de la calidad proteica de la biomasa autotrófica de Chlorella vulgaris. Alimento y Nutrición, 2: 8-123.

Quynh, VD.; Lavens, P.; Léger, P.; Tackaert, W.; Sorgeloos, P. 1988. Characterization of shrimp Artemia of cam Ramh Bay in Central Viethnam. Hydrobiology 157: 209-217.

Rodríguez-Canché, G.; Maldonado-Montiel, T.; Carrillo Navarro, L.

2006. Calidad biológica y bioquímica de la población de Artemia (Anostraca: Artemiidae) localizada en las salinas de Real de Salinas, Calkiní, Campeche, México. Rev. Biol. Trop. 54: 1283-1293,

Salgado, I.

2001. La Artemia y su cultivo en Perú. Universidad Nacional de Piura. Facultad de Ciencias Biológicas. Piura, Perú. $133 \mathrm{pp}$.

Sánchez-Estudillo, L.

2010. Alimento nutritivo, colorido y en movimiento: Los cultivos de apoyo en Acuicultura. Ciencia y Mar, 2011, 43: 55-60.

Sato, N.; Mallo, J.; Fenucci, J.

2004. Calidad de los quistes de Artemia persimilis (Piccinelli y Prosdocimi) (Crustacea: Branchiopoda) de diferentes zonas de Argentina, como alimento en acuicultura. Revista de Biología Marina y Oceanografía, 39: 79-92.

Soniraj, N.

2004. Effect of salinity on the life span and reproductive characteristics of brine shrimps in the salt pans at Tuticorin. Journal of the Marine Biological Association of India, 46: 133-140.

Sorgeloos, P.; Leger, P.; Tackaert, W.

1991. State of the art in larviculture the fish and shellfish. European Aquaculture society, special publication, Gen, Belgium, 15: 3-5.

Villamar, C.

2004. Protocolo para la cría de biomasa de Artemia adulta en raceways. AquaTIC, 21: 8-15.

Zamora, O.; Shpigel, M.

2006. Intensive mass production of Artemia in a recirculated system. Aquaculture, 255: 488-494. 